\title{
Antimycotic effectiveness against dermatophytes: comparison of two in vitro tests
}

\author{
Roberta Galuppi • Alessandra Gambarara • \\ Cristina Bonoli • Fabio Ostanello • \\ Maria Paola Tampieri
}

Published online: 19 May 2010

(C) Springer Science+Business Media B.V. 2010

\begin{abstract}
Seven antimycotic drugs (econazole, enilconazole, fluconazole, griseofulvin, itraconazole, ketoconazole, and miconazole) were tested against 36 dermatophyte strains (19 M. canis, 7 T. mentagrophytes, 5 M. gypseum, 2 M. cookei, 1 T. rubrum, 1 T. ajelloi, and 1 T. terrestre) isolated from animals, humans, and the environment. Two in vitro methods were compared: a micro-dilution test based on the CLSI M38-A method, and a disk-diffusion test. Fluconazole was not effective in vitro against the dermatophytes. Econazole and enilconazole were the most effective. Thirteen strains were griseofulvin-resistant. The correlation between the two methods was statistically significant for enilconazole, griseofulvin, itraconazole, and miconazole.
\end{abstract}

Keywords Antimycotic activity $\cdot$ Dermatophytes $\cdot$ In vitro tests

$\begin{array}{ll}\text { Abbreviations } \\ \text { MD } & \text { Microdilution method } \\ \text { DD } & \text { Disc diffusion method } \\ \text { DMSO } & \text { dimethylsulfoxide } \\ \text { PDA } & \text { potato dextrose agar } \\ \text { MIC } & \text { minimum inhibitory concentration }\end{array}$

\section{Introduction}

A reference method for the antifungal susceptibility testing of dermatophytes is not available. Some microdilution or disk diffusion systems are commercially available,

R. Galuppi $(\bowtie) \cdot$ A. Gambarara $\cdot$ C. Bonoli $\cdot$ F. Ostanello $\cdot$ M. P. Tampieri

Dipartimento di Sanità Pubblica Veterinaria e Patologia Animale,

Alma Mater Studiorum-Università di Bologna, Via Tolara di Sopra, 50, 40064 Ozzano Emilia, Bologna, Italy e-mail: roberta.galuppi@unibo.it

C. Bonoli

e-mail: cristina.bonoli3@unibo.it

F. Ostanello

e-mail: fabio.ostanello@unibo.it

M. P. Tampieri

e-mail: mariapaola.tampieri@unibo.it 
suitable mainly for yeasts and some filamentous fungi, such as Aspergillus fumigatus, Fusarium spp., and Rhizopus spp. The application of these tests to dermatophytes is problematic, due to, for example, the standardization of the inoculum and the final interpretation. The aim of this study was to evaluate the in vitro activity of certain antifungal drugs against different species of dermatophytes, using and comparing two methods: a microdilution method (MD) based on the document of the Clinical Laboratory Standard Institute CLSI M38-A, and a disk diffusion procedure (DD). The purpose was to establish a routine method to answer the demand of veterinary practitioners who want to verify whether the lack of response to treatment is linked to the resistance of dermatophytes involved rather than to host predisposing factors.

\section{Materials and methods}

Seven antifungal drugs (Econazole, Enilconazole, Fluconazole, Griseofulvin, Itraconazole, Ketoconazole, and Miconazole - Sigma Aldrich, St. Louis, MO, USA), commonly present in products used in the veterinary clinic, were tested against 36 strains of dermatophytes (19 Microsporum canis, 7 Trichophyton mentagrophytes, 5 M. gypseum, 2 M. cookei, 1 T. rubrum, 1 T. ajelloi, and 1 T. terrestre) isolated 1) from the skin of animals with or without lesions, 2) from the environment, and 3) from man (one case). The strains were spread on PDA (DIFCO) incubated at $30 \pm 2{ }^{\circ} \mathrm{C}$ for 14 days; for M. canis, three plates for each strain were prepared. The inocula were prepared by covering the PDA culture dishes with sterile saline solution, and the colonies were gently scraped with the tip of a Pasteur pipette. The resulting mixture of conidia and hyphal fragments was drawn and transferred to tubes containing five sterile glass balls, vortexed for 2-3 min, and kept at room temperature for $10 \mathrm{~min}$ (Singh et al. 2007). The optical density of the homogeneous supernatant suspensions was adjusted to level 2 of the McFarland scale. For each antifungal drug, a stock solution was prepared by dissolving the antimycotic powder in 100\% DMSO, except for Fluconazole, which was dissolved in sterile water (Jessup et al. 2000)

For the DD test, blank paper disks (Biolife) with a diameter of $6.0 \mathrm{~mm}$ were impregnated with $20 \mu \mathrm{L}$ of each concentration of stock solution (Table 1) or with $20 \mu \mathrm{L}$ DMSO. The disks were allowed to dry at room temperature. Petri dishes containing RPMI 1640 medium with L-glutamine (Sigma) buffered with $0.165 \mathrm{M}$ MOPS (Sigma) and $15 \mathrm{~g} / \mathrm{L}$ Bacto agar (DIFCO) (RPMI agar) were inoculated by dipping a sterile cotton swab into the inoculum suspensions and streaming the swab in four directions over the entire agar surface. The dishes were allowed to dry for 5 min before the disks loaded with drugs were applied. The

Table 1 Dilutions of the different antifungal drugs tested

\begin{tabular}{lllll}
\hline Antifungal drugs & Stock solution concentrations $(\mu \mathrm{g} / 20 \mu \mathrm{L})$ & \multicolumn{2}{l}{ Concentrations } & used for MD test $(\mu \mathrm{g} / \mathrm{mL})$ \\
\hline Enilconazole & 2 & (Fernandez-Torres et al. 2005) & 2 to 0.0019 & (Esteban et al. 2005) \\
Econazole & 2 & (Fernandez-Torres et al. 2005) & 2 to 0.0019 & (Esteban et al. 2005) \\
Miconazole & 10 & (Karaca and Koc 2004) & 4 to 0.008 & (Karaca and Koc 2004) \\
Ketoconazole & 15 & (Karaca and Koc 2004) & 4 to 0.008 & (Karaca and Koc 2004) \\
Fluconazole & 25 & (Singh et al. 2007) & 64 to 0.125 & (Singh et al. 2007) \\
Griseofulvin & 25 & (Karaca and Koc 2004) & 16 to 0.03 & (Singh et al. 2007) \\
Itraconazole & 10 & (Singh et al. 2007) & 8 to 0.05 & (Singh et al. 2007) \\
DMSO & whole & & 250 to 0.48 & \\
\hline
\end{tabular}


Table 2 In vitro activity of six drugs tested against 36 strains of dermatophytes determined by the DD and MD methods

\begin{tabular}{|c|c|c|c|c|}
\hline \multirow[t]{3}{*}{ Antifungal drug } & \multicolumn{2}{|l|}{ MD } & \multicolumn{2}{|l|}{ DD } \\
\hline & \multicolumn{2}{|l|}{$\mathrm{MIC}(\mu \mathrm{g} / \mathrm{mL})$} & \multicolumn{2}{|c|}{ Inhibition zone diameter (mm) } \\
\hline & Geometric mean & Range & Mean \pm std. dev. & Range \\
\hline Econazole & 0.21 & $0.03-1.0$ & $43.69 \pm 3.011$ & $0-80$ \\
\hline Enilconazole & 0.18 & $0.03-1.0$ & $50.47 \pm 3.842$ & $0-82$ \\
\hline Griseofulvin & 2.20 & $0.50-8.0$ & $41.19 \pm 3.815$ & $0-82$ \\
\hline Itraconazole & 0.96 & $0.06-4.0$ & $17.43 \pm 1.788$ & $0-38$ \\
\hline Ketoconazole & 0.76 & $0.06-1.0$ & $24.19 \pm 3.507$ & $0-62$ \\
\hline Miconazole & 0.29 & $0.03-1.0$ & $23.22 \pm 2.76$ & $0-50$ \\
\hline
\end{tabular}

$100 \%$ inhibition zone diameter was measured in mm using a ruler after 14 days of incubation at $30^{\circ} \mathrm{C}$. For the MD test, a 96-well microtiter plate (Sterilin) was used for each strain. RPMI broth (as above but without agar) was used. A column of the plates was used as a negative control (the culture medium only); another column was used as a positive control (100 $\mu \mathrm{L}$ medium and $100 \mu \mathrm{L}$ inoculum suspension). Ten different 2-fold dilutions of each drug and of DMSO were distributed in eight rows, and $100 \mu \mathrm{L}$ inoculum were added to each well. The plates were incubated at $30^{\circ} \mathrm{C}$ for 14 days. The minimum inhibitory concentration (MIC) was defined as the lowest concentration that produced $100 \%$ inhibition of growth. The correlation between the MD and DD tests were analyzed with the Pearson test, while the Mann-Whitney U test was used to evaluate the differences between the MIC of the geophylic vs. antropophylic/zoophylic dermatophytes.

\section{Results}

Fluconazole caused total inhibition of dermatophyte growth at the maximum concentration tested $(64 \mu \mathrm{g} / \mathrm{mL})$ only on three strains (one M. canis and two T. mentagrophytes) but was
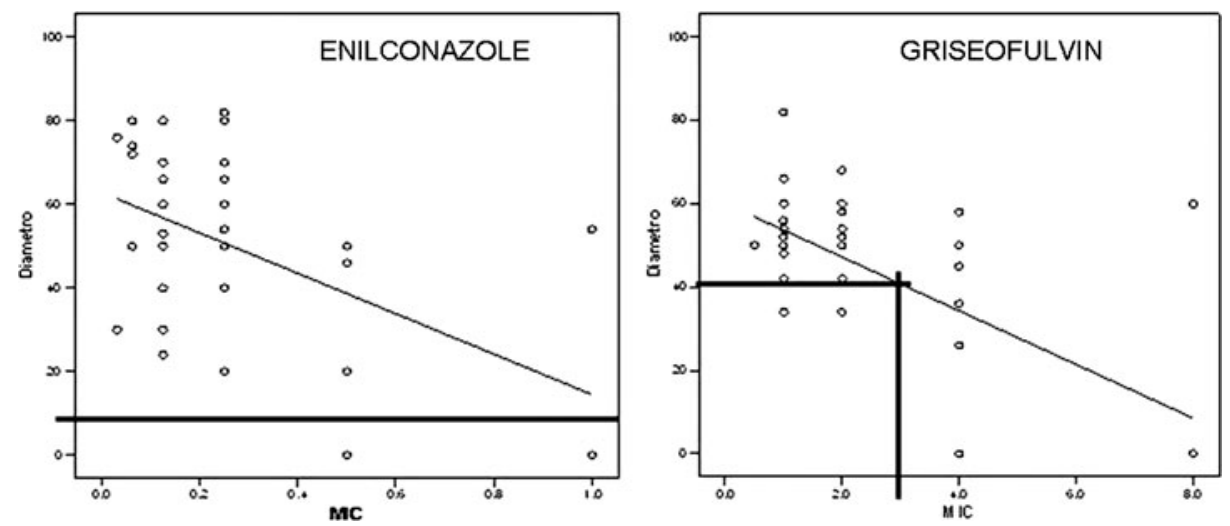

Fig. 1 Enilconazole and Griseofulvin disk zone diameters plotted against the MICs determined by the MD method 
ineffective against all other strains. In the DD test, these data were confirmed by the lack of an inhibition zone, except for one T. mentagrophytes strain, showing an inhibition halo of $18 \mathrm{~mm}$. Therefore, this drug was not considered further. The results obtained for the other antifungal drugs are shown in Table 2.

In the MD method, DMSO inhibited fungal growth up to the third dilution; for this reason, the first three dilutions of the drugs could be not indicative of real drug effects. As a whole, Econazole and Enilconazole were more effective than the other antimycotics tested, with MICs ranging from 0.03 to $1 \mu \mathrm{g} / \mathrm{mL}$. The geometric means of the MICs appear higher in geophylic vs. antropophylic/zoophylic strains: the Mann-Whitney U test showed statistically significant differences for Griseofulvin and Enilconazole. In the DD method, Econazole and Enilconazole resulted in greater inhibition zone diameters than the other drugs, despite their lowest concentrations $(2 \mu \mathrm{g})$ on the disk. When comparing the inhibition zone diameter and the MICs with the Pearson's test, statistically significant results were observed for Enilconazole, Griseofulvin, Itraconazole, and Miconazole.

Considering the regression lines obtained, we can affirm that for routine DD antimycogram, we considered, as effective, an inhibition zone diameter greater than or equal to $4 \mathrm{~mm}$ for Griseofulvin and greater than or equal to $18 \mathrm{~mm}$ for Enilconazole (Fig. 1).

\section{Discussion}

Fluconazole was considered ineffective against dermatophytes in accordance with other authors (Barros et al. 2007); indeed, the strains with MICs greater than or equal to $64 \mu \mathrm{g} / \mathrm{mL}$ are considered resistant (Gonçalves Krakhecke et al. 2005; Santos and Hamdan 2007). According to Randhawa (2006), high concentrations of DMSO, usually used as a drug diluent in antifungal in vitro tests, can inhibit fungal growth. For Griseofulfin, a MIC of $3 \mu \mathrm{g} / \mathrm{mL}$ was considered a limit of effectiveness (Scholz and Meinhof 1991). The mean MIC value was within the efficacy range of this drug $(2.2 \mu \mathrm{g} / \mathrm{mL})$, but 13 strains $(1 \mathrm{M}$. canis, 2 T. mentagrophytes, $5 \mathrm{M}$. gypseum, $2 \mathrm{M}$. cookie, and the T. rubrum, T. terrestre and T. ajelloi strains) were resistant, with MICs ranging from 4 to $8 \mu \mathrm{g} / \mathrm{mL}$. In the literature, cases of dermatophyte resistance to Griseofulvin are described for T. rubrum (Korthing et al. 1995). With the DD method, it was not possible to evaluate the minimum inhibition diameter for Miconazole and Itraconazole, as the MIC described by some authors as indicative of drug resistance in different mycetes are higher than the concentrations of the drug in the paper disk. Nevertheless, we conclude that, with some adjustment, the DD method could be used as a screening procedure to evaluate the in vitro sensitivity of dermatophytes to commonly used antimycotics in clinical practice.

\section{References}

Barros MES, Santos DA, Hamdan JS (2007) Evaluation of susceptibility of Tricophyton mentagrophytes and Tricophyton rubrum clinical isolates to antifungal drugs using a modified CLSI microdiluition method (M38-A). J Med Microbiol 56:514-518

Esteban A, Abarca ML, Cabanes FJ (2005) Comparison of disk diffusion method and broth microdiluition method for antifungal susceptibility testing of dermatophytes. Med Mycol 43:61-66

Fernandez-Torres B, Inza I, Guarro J (2005) Evaluation of disk diffusion method for determining eberconazole susceptibility of dermatophytes and influence of culture medium. Antimicrob Agents Chemother 49:2116-2118 
Gonçalves Krakhecke A, Euripedes A, Ferreira JC, Candido RC (2005) In vitro susceptibility testing of Microsporum gypseum isolated from healthy cattle and soil against itraconazole, terbinafine, fluconazole, and topical veterinarian drugs. Mycopathologia 159:377-380

Jessup CJ, Warner J, Isham N, Hasan I, Ghannoum MA (2000) Antifungal susceptibility testing of dermatophytes: Establishing a medium for inducing conidial growth and evaluation of susceptibility of clinical isolates. J Clin Microbiol, 38(1):341-344

Karaca N, Koc AN (2004) In vitro susceptibility testing of dermatophytes: Comparison of disk diffusion and reference broth diluition methods. Diagn Microbiol Infect Dis 48:259-264

Korthing HC, Ollert M, Abeck D and the German collaborative dermatophyte drug susceptibility Study Group (1995) Result of German multicenter study of antimicrobial susceptibilities of Trichophyton rubrum and Trichophyton mentagrophytes strains causing Tinea unguium. Antimicrob Agents Chemother 39:1206-1208

Randhawa MA (2006) The effect of dimethylsulfoxide (DMSO) on the growth of dermatophytes. Nippon Ishinkin Gakkai Zasshi 47:313-318

Santos DA, Hamdan JS (2007) In vitro activities of four antifungal drugs against Trichophyton rubrum isolates exhibiting resistance to fluconazole. Mycoses 50:286-289

Scholz R, Meinhof W (1991) Susceptibility of Trichophyton rubrum to griseofulvin. Mycoses, 34: 411-414

Singh J, Zaman M, Gupta AK (2007) Evaluation of microdilution and disk diffusion methods for antifungal susceptibility testing of dermatophytes. Med Mycol 1:1-8 